\title{
Overview of the ITER-like Wall Project
}

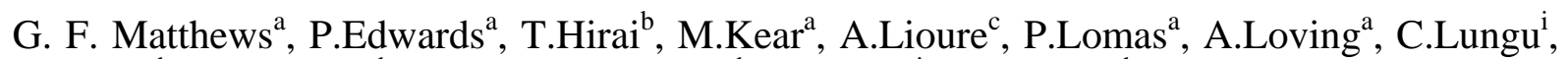
H.Maier ${ }^{\mathrm{d}}$, Ph.Mertens ${ }^{\mathrm{b}}$, D.Neilson ${ }^{\mathrm{a}}$, R.Neu ${ }^{\mathrm{d}}$, J.Pamela ${ }^{\mathrm{j}}$, V.Philipps ${ }^{\mathrm{b}}$, G.Piazza ${ }^{\mathrm{c}}$, V.Riccardo ${ }^{\mathrm{a}}$, M.Rubel $^{\mathrm{e}}$, C.Ruset ${ }^{\mathrm{i}}$, E.Villedieu ${ }^{\mathrm{f}}, \mathrm{M}$.Way ${ }^{\mathrm{a}}$ on behalf of the ITER-like Wall Project Team ${ }^{\mathrm{a}-\mathrm{k}}$

${ }^{a}$ Euratom/UKAEA Fusion Association, Culham Science Centre, Abingdon, UK

${ }^{b}$ Forschungszentrum Jülich, Euratom Association, Jülich, Germany

${ }^{c}$ EFDA-Close Support Unit, Culham Science Centre, Abingdon, UK

${ }^{d}$ Max-Planck-Institut für Plasmaphysik, EURATOM Association, 85748 Garching, Germany

${ }^{e}$ Alfvén Laboratory, Royal Inst. Technology (KTH), Assoc. EURATOM-VR,100 44 Stockholm, Sweden

${ }^{f}$ Association Euratom-CEA, Cadarache, DSM/DRFC, Saint Paul Les Durance, France

${ }^{g}$ Association Euratom-TEKES, VTT Processes, Finland

${ }^{h}$ Association Euratom-ENEA, Frascati, Italy

'Nat. Inst. for Laser, Plasma and Radiation Physics, Association Euratom-MEdC, Bucharest, Romania ${ }^{j}$ EFDA-Close Support Unit, 85748 Garching, Germany

${ }^{k}$ Association Euratom/SCK-CEN, Boeretang 200, 2400 Mol, Belgium

EURATOM/UKAEA Fusion Association, Abingdon, OX14 3DB, UK

\begin{abstract}
Work is in progress to completely replace, in 2008/9, the existing JET CFC tiles with a configuration of plasma facing materials consistent with the ITER design. The ITER-like Wall (ILW) will be created with a combination of beryllium (Be), tungsten (W), W-Coated CFC and Be-Coated inconel tiles, with the material depending on the local anticipated heat flux and geometry. It is part of an integrated package of JET enhancements whose aim is to develop an understanding of the ITER materials issues and develop the techniques required to operate with inductive and advanced scenarios as close as possible to ITER parameters. Over 4000 tiles will be replaced and the ILW will accommodate additional heating up to at least 50 MW for $10 \mathrm{~s}$. This paper describes the scientific background to the project, the technical objectives, the material configuration selected, the R\&D behind the practical realisation of the objectives and the generic problems associated with the Be tiles (power handling capacity and disruption induced eddy currents). One of the objectives is to maintain or improve the existing CFC tile power handling performance which has been achieved in most cases by hiding bolt holes, optimising tile size and profile and introducing castellations on plasma facing surfaces.
\end{abstract}

Keywords: JET, ITER-like Wall, beryllium wall, tungsten divertor 


\section{Introduction}

Starting in July 2004 the concepts were developed for an integrated set of new enhancements (EP2) to make JET a test-bed for developing ITER scenarios. Key elements of this package were an upgrade of the neutral beam heating power to give JET a total combined heating power of around 50MW. A high frequency pellet launcher for ELM control, a package of relevant diagnostic upgrades and finally an ITER-like Wall [Pamela2006a,b]. In October 2004 an international workshop including representatives of the ITER Project was held at JET to discuss options for an ITER-like Wall Project (ILW). The recommendation made at this workshop was that a project should be launched with the aim of testing a full beryllium wall and full tungsten divertor, Fig. 1, rather than the ITER reference materials configuration which includes CFC areas at the inner and outer strike zones. There were three principal reasons for this decision:

i. A full tungsten divertor is regarded as a backup solution for ITER if carbon generated at the strike zones leads to unacceptable tritium retention.

ii. An all metal machine places a clear boundary on what is possible without the complication of a carbon source which might make interpretation difficult.

iii. If JET were to start operation with uncoated CFC tiles at the strike zones then there is a risk of widespread carbon contamination that would make a subsequent move an all metal configuration difficult to prepare due to the need to remove all residual carbon contamination from the vessel. 


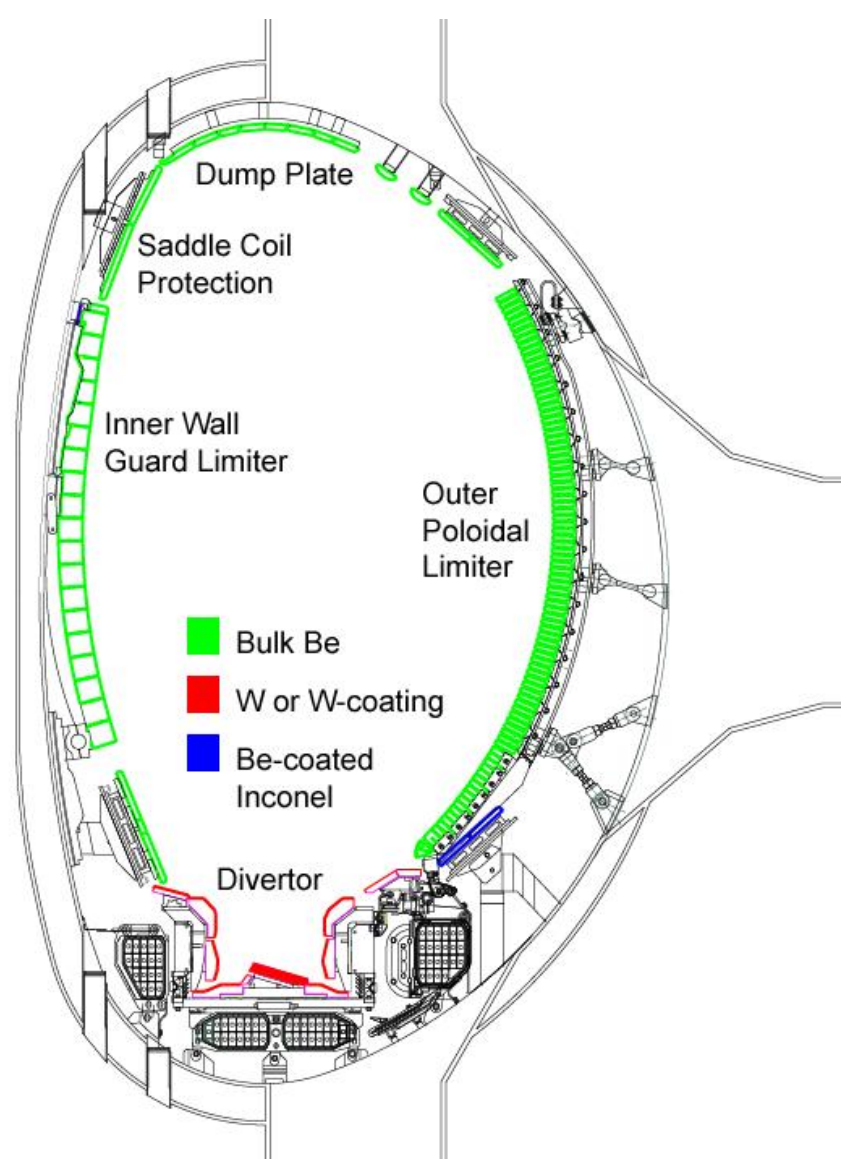

Fig. 1 JET materials configuration selected for the ITER-like Wall Project

In April 2005 the package of enhancements in the EP2 programme, including ILW, received final approval. This will provide the basis for an integrated approach to preparing for ITER operation. The principal elements of EP2 are the new wall and diagnostics required to exploit it, a neutral beam upgrade to push JET parameters into a more ITER-relevant regime and a high frequency pellet injector for ELM control [Pamela2006a,b]. After approval, the ILW project then had until May 2006 to build up the Engineering, Installation preparation and R\&D teams with the objective of designing a new wall for JET and preparing all call for tender documentation. This was a major undertaking given that the JET wall consists of more than 4000 CFC tiles of many different types which will have to be removed and replaced by remote handling. One year was also a challenge for performing all necessary R\&D on Be and $\mathrm{W}$-coating technologies and bulk $\mathrm{W}$ tile design. This required timescale also set limits on what was technically possible. 


\section{Scientific Objectives}

\subsection{ITER Context}

The current design for the ITER main wall consists of $700 \mathrm{~m}^{2}$ of actively cooled beryllium. This choice is driven by considerations of plasma performance and tritium retention. Beryllium is the lowest $\mathrm{Z}$ material in the periodic table offering good thermal and mechanical properties. It is therefore relatively benign in terms of plasma dilution and radiates power only from near the plasma edge which offers the prospect of a wide operating space in ITER without the risk of impurity accumulation and core radiation associated with high $\mathrm{Z}$ metals. Beryllium is also an oxygen getter which should help to keep the $Z_{\text {eff }}$ in ITER at an acceptable level. Finally, beryllium is expected to provide lower tritium retention compared to an all carbon wall (although the benefit may be reduced when impurities are present).

At the strike point regions, ITER will have $50 \mathrm{~m}^{2}$ of actively cooled CFC tiles. This material offers excellent heat shock resistance without a liquid phase and is considered to have the best chance of surviving the large thermal transients due to ELMs and disruptions which are possible in ITER. However, the big concern about using any CFC in ITER is the potential for tritium retention in hydrocarbon deposits that may be generated in remote areas of the divertor. The rest of the divertor (baffles and dome) are tungsten. Like other high Z materials tungsten has a low erosion rate at low ion impact energies but also has excellent power handling capabilities and low tritium retention.

The ITER reference materials choice is thus a compromise based on experience of the individual materials in many different tokamaks but the actual combination and our ability to work strictly within the limits imposed by these materials has never been tested. The primary aim of the ITER-like Wall Project is to improve our understanding of the many potential problems outlined in subsequent sections for the most relevant conditions and to gain experience in JET so that ITER will be more adequately prepared for operation in all the reference scenarios [Pamela2006a].

\subsection{Issues for a beryllium main chamber wall}

The main issues that will be addressed by the beryllium wall in JET are:

a) Erosion and migration of the main wall material in ITER-relevant scenarios. This will be diagnosed pulse to pulse by improved spectroscopic diagnostics and by postmortem analysis using special marker tiles which are under development 
[C.Lungu2006] and will consist of a high Z marker layer under an 8-10 $\mu$ m thick layer of beryllium.

b) Behaviour of molten beryllium and impact on operation and wall lifetime. A full range of diagnostics: visible and IR cameras, spectroscopy and post-mortem analysis are necessary to investigate this complex problem.

c) Fuel (deuterium) retention in beryllium tiles (surface and bulk) and in codeposited layers. This investigation relies on post-mortem analysis.

d) Interaction of beryllium with background oxygen. This includes more extreme exposure such as leaks and venting. The key diagnostic tools in this case are assessment of operational impact, spectroscopy and post-mortem analysis.

e) Wall conditioning and machine start-up. This is relevant after installation or intervention and could be carried out at an ITER-relevant baking temperature $\left(200^{\circ} \mathrm{C}\right)$, without the use of beryllium evaporation.

\subsection{Issues for an all tungsten divertor}

The main issues that will be addressed by the tungsten divertor are:

a) Compatibility with ITER scenarios. One key element of this is the study of tungsten sources and accumulation in the core of the plasma which is a major concern for Advanced Tokamak (AT) scenarios. Another is the control of steady state divertor power load in the absence of carbon radiation which is an issue for both inductive and AT scenarios.

b) Tungsten material issues. This includes: determination of fatigue life under repetitive transient power loads (ELMs), alloying of beryllium with tungsten which reduces the melting point and the behaviour of tungsten melt layers. To study these issues, implementation of a bulk tungsten tile in a high heat flux area is essential. Diagnosis would be by high resolution inter-shot photography and post-mortem surface analysis.

c) Tritium retention including the migration of beryllium and residual carbon into gaps. With the existing CFC wall about fourteen times as much carbon as beryllium migrates to the divertor but excess carbon is eroded from the deposits by hydrocarbon chemistry. This leaves a beryllium carbide layer on the plasma facing surface and deuterium/tritium rich hydrocarbon layers in remote areas [Coad2001]. With a beryllium wall, the deposition on the tungsten surface will be beryllium dominated. A key issue is whether there is onward migration of surface beryllium, as reported in PISCES [Doerner2006], or whether it merely accumulates on plasma facing areas. 
This question has a big impact on what techniques will be required to recover tritium retained in ITER.

\subsection{Programmatic implications}

Plasma control and machine protection, see section 4, will be key elements of any programme to exploit the ITER-like Wall. It is also clearly desirable to delay the study of operation with more extreme melt damage to wall surfaces until the end of the programme. As can be seen from the list of scientific issues above, to obtain definitive answers to many of the scientific questions surrounding the wall requires post-mortem surface analysis of tiles and of specially designed marker samples. The programme will therefore have to be structured to allow for regular fast interventions for sample removal and replacement. A preliminary analysis suggests that this is consistent with other constraints on JET operations.

\section{Engineering of the ITER-like Wall}

\subsection{Technical objectives}

The technical objectives for the new wall are driven by the desire to preserve and improve as best we can the performance of the wall whilst meeting a very tight procurement schedule. More specifically, the objectives are to install bulk beryllium tiles in main chamber areas of highest plasma-wall interaction and full tungsten divertor consistent with:

a) Elimination of all significant sources of carbon.

b) Eddy current forces consistent with the strength of existing support structures and JET design rules $\left(\mathrm{I}_{\mathrm{p}}=6 \mathrm{MA}, \mathrm{B}_{\mathrm{T}}=4 \mathrm{~T}\right)$. This has a major design impact because the resistivity of Be at $200^{\circ} \mathrm{C}$ is $0.08 \mu \Omega \mathrm{m}$ compared to $10 \mu \Omega \mathrm{m}$ for CFC.

c) Limiter energy and power handling equal to or better than the current CFC design. This is challenging due to relatively low melting point of $\mathrm{Be}\left(1289^{\circ} \mathrm{C}\right)$ compared to the ablation limit for carbon at $\sim 2700^{\circ} \mathrm{C}$ (depends on the vapour pressure deemed acceptable).

d) A grade of beryllium with minimum risk of cracking / fragmentation.

e) Remote handling compatible design which includes the requirement that tile assemblies for installation on the main wall area weigh less than $10 \mathrm{~kg}$ and in the divertor less than $100 \mathrm{~kg}$ including tooling.

f) Neutral beam shine-through energy handling $>60 \mathrm{MJm}^{\mathrm{b}}$ which is nearly double the present value to meet the needs of AT scenarios. 
g) Designs compatible with a hard limit of one year to complete all R\&D and the design work necessary for the issue of all calls for tender.

In addition to the above, there is an objective for a shutdown time for removal and replacement of all wall and divertor components of 46 weeks or less. This is not feasible using the existing remote handling equipment, nor is it compatible with a mainly manual intervention and so the installation strand of the project includes provision of a second long remote handling boom. Instead of delivering components one at a time at one particular port as at present, task modules will be delivered close to the work area which will contain a whole set of components and related tools. This is predicted to substantially increase the efficiency of remote handling operations.

\subsection{Research and Development Overview}

In order to meet the scientific and technical objectives for ILW it was evident from the start that it would be necessary to initiate a number of critical R\&D tasks. The first of these was to evaluate a wide range of industrial technologies for tungsten coating of 2D carbon fibre composite tiles with thermo-mechanical properties like those of the existing JET tiles in order to minimise the amount of redesign required. The anisotropy in the thermal expansion of this material has made this quite challenging but one $200 \mu \mathrm{m}$ VPS and a $10 \mu \mathrm{m}$ CSMII process fully met the test requirement and were selected for implementation. This work is described in a companion paper [Neu2006] and [Maier2006]. The second key R\&D task was to develop an inertially cooled bulk tungsten tile design to replace the toroidal belt of tiles at the outer strike zone which is both a region of net erosion and the area of highest heat flux. This work is described in detail in a companion paper [Mertens2006, Hirai2006a,b]. The third R\&D task, which is still ongoing, was to develop a high Z marker with beryllium overlayer so that erosion of beryllium tiles in the range $0-10 \mu \mathrm{m}$ can be measured. Changes larger than this are more readily determined by direct surface measurement. The fourth and final R\&D task was to develop 8-10 $\mu \mathrm{m}$ beryllium coatings for recessed inconel tiles and tile carriers which are subjected mainly to charge exchange neutral fluxes. The purpose is to maximise the area of beryllium at acceptable cost. Tests of erosion and thermal performance [Hirai2006c] suggest that the lifetime of these coatings will be sufficient to meet the programmatic goals. Further details of these last two tasks are given in a companion paper [Lungu2006]. 


\subsection{Tungsten divertor material configuration}

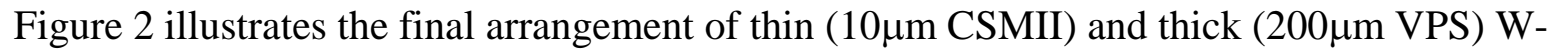
coatings and bulk W selected for use in the divertor. The reason for choosing the thick coating in the outer divertor was that $3 \mu \mathrm{m}$ thick tungsten marker stripes, which were exposed to $~ 80,000$ s of JET divertor plasma operation, were totally eroded in some places [Mayer2006]. This result is consistent with ERO simulations which predict peak erosion of order $10 \mu \mathrm{m}$ which would be incompatible with thin coatings. More surprising and not yet conclusively explained was a high level of erosion on the horizontal face at the top of tile 8 where the ion flux is expected to be low. Flux maps of typical magnetic configurations suggests that tiles 5a, B and C are not normally expected to receive significant plasma fluxes and so thin coatings have been selected. The choice of a thick coating at the inner divertor is less obvious since the inner divertor is expected to be a deposition zone and the $3 \mu \mathrm{m}$ thick tungsten marker stripes in this area were intact but buried. The decision was however driven by a concern that when the deposited material is primarily beryllium it would be more difficult to study alloying of beryllium deposits with a tungsten substrate and also because the thin coating had proven less resilient to ELM-like heat loads in tests carried out with the JUDITH-1 high heat flux facility.

The high erosion rate expected on tile 5 in ITER-like high triangularity plasma configurations and the risk of melting due to transients were the main reason for choosing a concept based on bulk material. If melting were to occur the effect of surface tension means that tungsten coatings tend to bead up whereas melt layers on bulk material in the tests carried out in TEXTOR show a much more benign behaviour so including a bulk $\mathrm{W}$ tile reduces the risk of irreversible damage in the highest power density area of the divertor. In order to minimise the electromagnetic loads, a lamellae design was selected. It breaks the loops that would otherwise be available to eddy current flow and provides a vertical path to the base plate for the halo current. More details can be found in [Mertens, 2006]. 


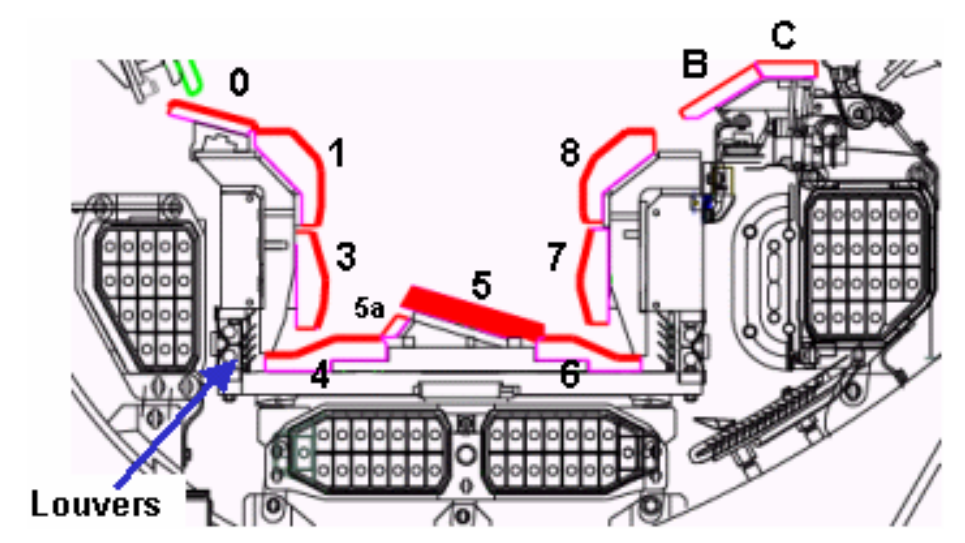

Figure 2. Material configuration selected for the all tungsten divertor. Tiles 0,1,3,4,6,7,8 will

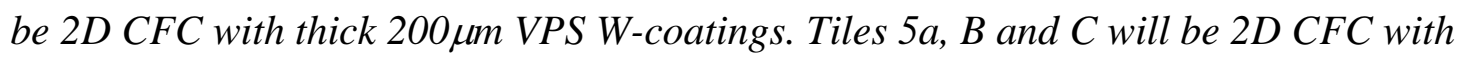
thin10 m CSMII W-coatings. Tile 5, the high heat flux area for ITER-like configurations will be a bulk tungsten design.

A major concern with coatings is the need to ensure that there are no manufacturing defects which might lead to lack of adhesion and loss of thermal contact. To try to tackle this, R\&D was carried out on IR based methods using high power flash-lamps or lock-in thermography to try to detect defects prior to heat flux testing. The results were however disappointing in that defects could appear during heat flux tests which were not apparent in the pre-test. In consequence a task has been launched to use the JUDITH-2 high heat flux electron beam facility at Forschungzentrum Juelich to test all production W-coated divertor tiles with moderate but JET relevant thermal loads. A similar strategy is also being applied to thin coatings on the main chamber wall but in this case up to $10 \%$ of tiles will be tested in the GLADIS neutral beam test facility at IPP Garching.

\subsection{Material configuration of the main wall}

The ITER reference design has a more or less continuous actively cooled beryllium wall surface. The technical objectives for the JET ITER-like wall project mean that this ideal has to be compromised in a number of ways. Firstly, an actively cooled design was not feasible given constraints on resources and existing JET support structures and secondly, the existing JET wall relies on a series of discrete poloidal limiters at the inner and outer walls. However, the more fundamental similarity to ITER is that solid beryllium is used at the primary contact points between the plasma and the main wall. The area of greatest compromise to our ideal 
materials experiment has been made in trying to meet the objective to increase the energy handling of the inner wall for neutral beam shine-through. This requirement is driven by the needs of Advanced Tokamak (AT) scenarios which are currently formed at low density and thus high shine-through power and maintained non-inductively by bootstrap current and neutral beam current drive which is most efficient at lower densities and so high shinethrough fractions. Increasing the density of AT scenarios in the run-up to the ITER-like Wall experiment is a key goal but the technical requirement stands. The difficulty it creates is that the normal and tangential banks impinge on significant areas of the inner wall and if bulk beryllium were used in these areas, the limits on shine-through power would have to be lowered from current values. In order to meet the technical objective, CFC tiles with thin tungsten coatings will be used in these areas which are similar to the existing design but with higher temperature internal metallic components. To minimise interactions with the plasma, all main chamber tungsten coated CFC components are recessed at least $1 \mathrm{~cm}$ behind the limiter radius and in most cases at least $2.5 \mathrm{~cm}$. This puts all main chamber CFC which has thin tungsten coatings in areas which, from past experience, we know are either deposition zones or areas of low erosion rate. Erosion due to sputtering by the neutral beam shinethrough power $\left(140 \mathrm{KeV} \mathrm{D}^{+}\right)$is not significant but the $\mathrm{W}$ source of around $2 \times 10^{17}$ atoms $/ \mathrm{s} / \mathrm{MW}$ may cause impurity problems for AT scenarios where there is a strong internal transport barrier. If the sputtered tungsten from the inner wall seriously impacts plasma operation, it would be possible to use the beryllium evaporators, which are positioned directly opposite the shine-through areas, to add a layer of beryllium on top of the tungsten. Figure 3 shows the inner wall material map. Fewer tungsten coated tiles are required on the outer wall but equivalent problems have had to be addressed and similar solutions adopted. For example, the right hand frame of figure 4 shows the magnetic pickup coils which run alongside some of the Wide Outer Poloidal Limiters. A beryllium coil cover was not an option due to the time it would take the field to penetrate and so a $10 \mu \mathrm{m}$ W-coated CFC cover will be used instead and this is regarded as acceptable given its recessed location. 


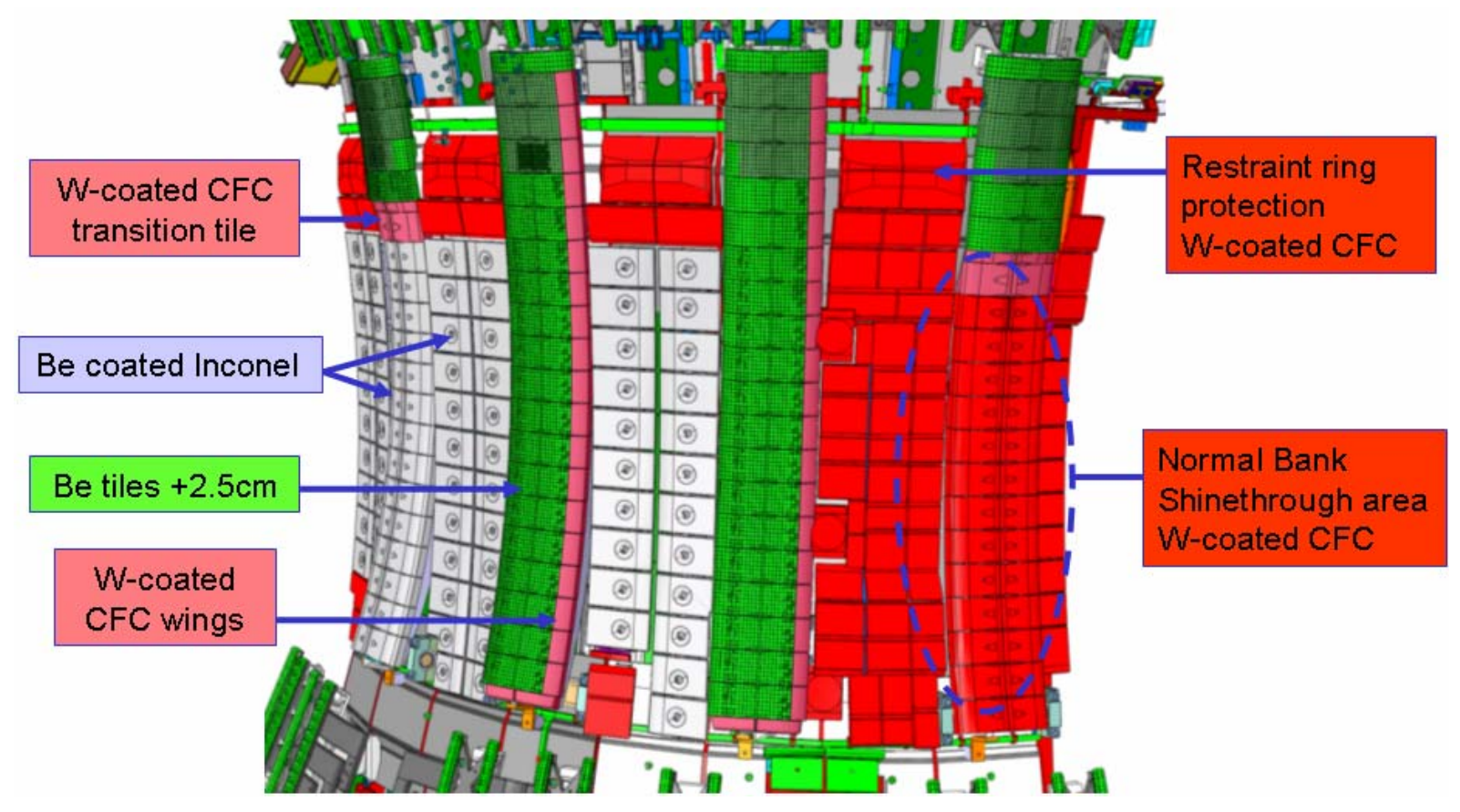

Figure 3. Map of the inner wall material configuration. The tungsten areas are mainly driven by the technical objective to increase the neutral beam shine-through energy handling and maintain the shine-through power handling. The beryllium tiles are $2.5 \mathrm{~cm}$ forward of the $W$ coated CFC tiles in the normal bank shinethrough area.

The reference material Be for ITER is Brush Wellman S65C VHP (vacuum hot pressed) which has the best combination of thermal stress induced crack resistance and other mechanical properties of any type of beryllium [Watson, 1997]. For the ITER-like Wall we have selected S65J HIP (hot isostatic pressed) material which produces stronger and more isotropic material properties and is better suited to producing large blocks. An additional factor was that JET had 4 tons of beryllium components (mostly S65C) which are providing the feedstock for production of the new blocks.

\subsection{Beryllium tile design}

Figure 4 shows the layout of the wide outer poloidal limiter along with an exploded view of one of the beryllium tile assemblies which serves as a good example for the design features that have allowed us to meet the technical objectives for the wall [Thompson2006]. 


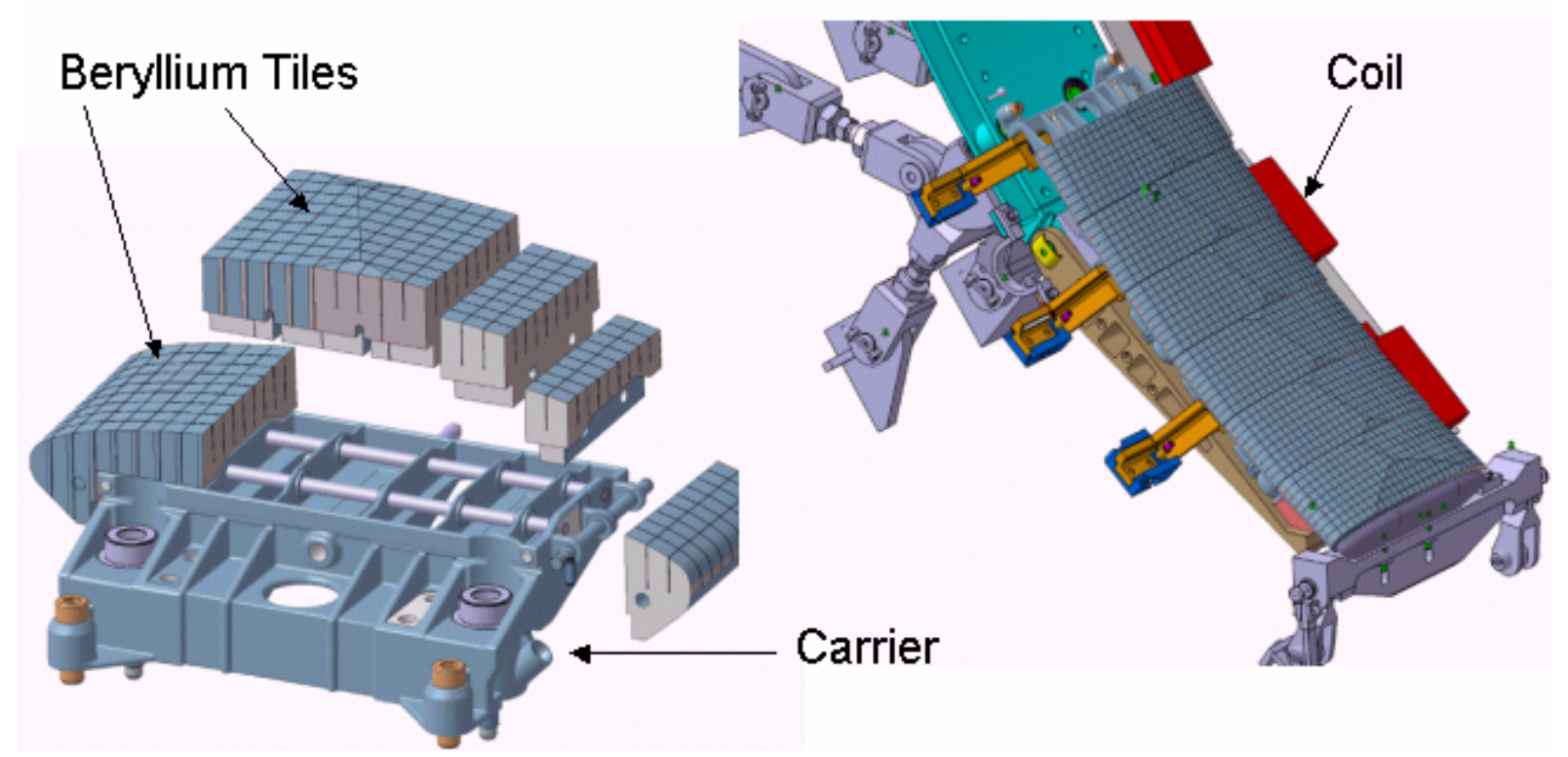

Figure 4. Wide outer poloidal limiter assembly(right) and an exploded view of one of the tile carriers (left).

\subsubsection{Power handling}

At $40 \mathrm{~mm}$ typical thickness, the tiles are "thermally thick" for 10s pulses. Surface castellations and kinematic restraints will be used to minimise thermally induced stresses. As the thermal flux arrives along near toroidal directions, the design keeps the exposed depth of poloidally running edges to low levels: down to $40 \mu \mathrm{m}$ in the most severe positions. This limit strongly constrains the dimensions of the castellation grooves and the location of the cuts described below. The plasma facing surfaces of the tiles are shaped to optimise power handling for a range of power decay lengths and a wide range of plasma configurations $\left(\mathrm{q}_{95}=\right.$ 2.2-11.5) [Nunes2006]. Significant improvements over the current CFC tiles have been obtained by eliminating bolt holes, increasing the poloidal extent of the tiles and interlocking of tile carriers to control toroidal gaps. This has in most cases recovered the factor 2-3 needed to maintain overall thermal performance.

The power handling of the tiles is driven by the need to (a) avoid surface melting and (b) reduce thermally induced stresses to give an adequate fatigue life. For the typical JET cycle of pulses lasting $10 \mathrm{~s}$ at 20 minute intervals, the melting limit combined with the thermal conductivity and capacity of Be gives an upper bound to the power handling (measured normal to the tile) of about $6 \mathrm{MW} / \mathrm{m}^{2}$ and a minimum thickness of about $40 \mathrm{~mm}$. The power level of $6 \mathrm{MW} / \mathrm{m}^{2}$ would apply in cold start cases where the pre-pulse tile temperature is 200 ${ }^{\circ} \mathrm{C}$. Between pulses, the tiles cool by radiation exchange within the torus and so the tile bulk 
temperature will rise after a number of pulses to the hot start case of about $400{ }^{\circ} \mathrm{C}$. This will reduce the power handling capacity to about $4 \mathrm{MW} / \mathrm{m}^{2}$.

The fatigue life issue is much more complicated to quantify due to a number of factors. In particular, there are only limited data on relevant mechanical properties of Be at elevated temperatures, and so the aim has been to minimise thermally induced stress as far as practical in order to maximise the fatigue life. The thermal stresses depend on the temperature profile and the degree of constraint in the tiles. At the tile level these constraints have been eliminated by using kinematic supports which allow the tile to both expand and bow in response to the thermal expansion. At the local level, castellations have been introduced to further relieve the constraints, and these have been sized following an extensive program of coupled thermal and mechanical analyses using FE (Finite Elements) using the ANSYS and ABAQUS codes. The studies concluded that the optimum castellation size is $6 \mathrm{~mm} \times 6 \mathrm{~mm}$ area $\times 16 \mathrm{~mm}$ deep with a keyhole profile as this gave similar thermal and mechanical performance, but for reasons of fabrication cost the area has been increased to $12 \mathrm{~mm} \times 12$ $\mathrm{mm}$ and the keyhole replaced by a half-round in most cases.

\subsubsection{Hot spots at steps and edges}

The requirement for castellations and slicing of the tiles (to reduce eddy currents as described below) results in small exposed edges on the toroidally facing surfaces. With typical angles of attack to the normal face of $1-5 \mathrm{deg}$, the heat flux on the exposed edges is $45-230 \mathrm{MW} / \mathrm{m}^{2}$ and the edge exposure must be minimised to avoid melting at hot spots. Detailed FE and analytical models have provided results for sensitivity studies to arrive at design limits for the exposed depths (and the associated toroidal gaps). Figure 5 shows the calculated maximum temperature on an exposed edge for different field line angles and a fixed power load of 4 $\mathrm{MW} / \mathrm{m}^{2}$ at the front surface of the tile. Shallow field line angles therefore imply a higher parallel power density and so higher edge heating. 
Face and Exposed Edge Heating (Step)

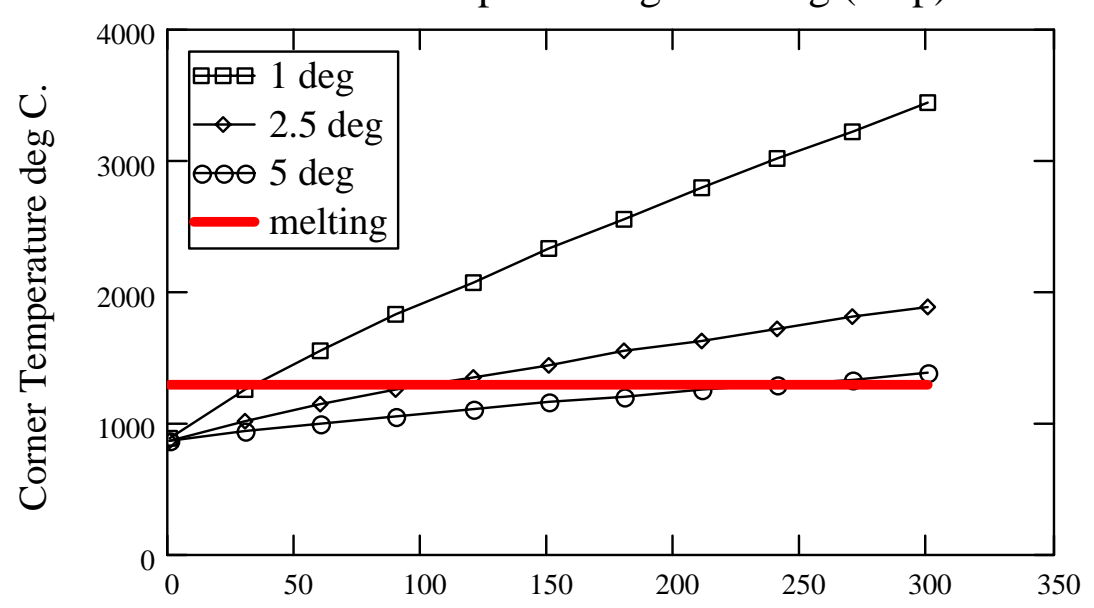

Edge exposure length in microns

Figure 5. Calculated peak temperature on an exposed edge for a fixed power density to the front face of the tile. Different angles are shown. Edge exposures up to $40 \mu \mathrm{m}$ are allowed by the ILW design rules.

As the exposures reduce, the results converge on a temperature of about $840{ }^{\circ} \mathrm{C}$ (for 4 $\mathrm{MW} / \mathrm{m}^{2}$ ) which is the case for face heating only. Although the change with exposure is fairly small, the proximity to melting means that only small exposures can be tolerated and a value of $40 \mu \mathrm{m}$ has been set to be consistent with the $4 \mathrm{MW} / \mathrm{m}^{2}$ and 1 degree field line angle case and leave some margin. In consequence, the tile employs a large block in the central region where the heat flux is high. This block contains only castellations which have small exposures. The slices, which are needed to reduce eddy currents but which cannot be assembled with accuracy sufficient to ensure exposed edges are $<40$ micron, are banished to the outer regions of the tile where the heat flux is lower and so greater exposures can be tolerated. Shingling is also used to hide edges between adjoining blocks where tolerances will be worse than for internal castellations.

\subsubsection{Eddy and halo current forces}

During disruptions, Be tiles are subjected to eddy current torques due to large changes in magnetic field. The ILW Be tiles will manage these torques via a combination of the castellations, along with cuts which will interrupt the eddy current loops. The cuts result in the division of the tiles into several slices which require inconel carrier "toast racks" for support. The tile assembly must also withstand e/m forces due to halo currents which, during disruptions, pass between plasma and vacuum vessel via the tiles. 
The carrier designs are driven by their interfaces with the sliced Be, the existing limiter beams, the requirement for remote handling and the limited space available. The requirements result in the complex geometry shown above, which together with the large number required ( 1000) means that casting is the appropriate manufacturing route. This limits the choice of suitable materials to: inconel 600 air-cast, vacuum-cast 625 and vacuum-cast 713LC in order of preference based on cost and ease of manufacture. Because the material choice depends on the strength requirement, the complexity of the geometry and loading means that detailed FE analysis was needed for the assessment.

\section{Operational Issues}

Current tokamaks including JET which have inertially cooled carbon or CFC plasma facing components and relatively low stored energy compared to ITER, enjoy a situation where the plasma facing components are almost indestructible because the carbon bloom is a relatively benign thermal limit which may do nothing more than round off the offending edges. This will of course no longer be true for the beryllium wall and tungsten divertor. Reaching these limits will not necessarily create serious problems for the bulk beryllium tiles or bulk tungsten divertor tiles. There is evidence from tests in TEXTOR that exposed edges can adopt a more optimal shape after melting [Mertens2006] but we cannot be sure that the same will be true in JET. The tungsten coated tiles are more of a concern because once melted the tungsten forms droplets which will not re-wet the CFC surface due to the effect of surface tension. It is therefore a necessity to develop techniques to minimise the risk of unscheduled melting of the wall or divertor which could be caused by: a high steady state power density, neutral beam shine-through power, hot spots associated with special RF heating effects by LH or ICRH, transient power loads from ELMs and disruptions.

To maximise machine performance and approach most closely ITER conditions, JET will have to make full use of the heating (40-50MW) and plasma current (5-6MA) capability. This will mean operating well beyond the point at which, if control is lost, serious damage to the ITER-like Wall can result. There are a number of ways in which these risks can be minimised:

a) A phased programme in which performance is built up slowly and carefully.

b) More strict JET Operating Instructions (JOIs) which limit the operational space that the JET machine is permitted to explore or sets strict conditions on entering dangerous parts of operational space. A main chamber thermocouple array is being installed as part of the ILW project and will help set the limits more accurately than in the past. 
c) Real time control schemes to keep parameters within acceptable limits for example by controlling plasma radiation levels or ELM frequency. If control fails to keep plasmas within acceptable limits then protection systems are required to reliably detect a problem and shut down the plasma by turning off the heating, firing the disruption mitigation valve or whatever is the safest plasma termination method.

\section{Conclusion}

The approval of the ITER-like Wall Project and the progressive approach of ASDEXUpgrade towards a full tungsten wall [Neu2006b] are a demonstration of European commitment to tackling the wall materials issues which are critical for the medium and long term future of fusion. The full tungsten wall is focused on DEMO relevance whilst the ITERlike Wall aims to demonstrate the greater flexibility which led to the material combination selected for ITER. Any wall material in a next step device such as ITER or DEMO will only survive if an integrated and highly validated approach is taken to material selection, scenario development, control and machine protection. Although experiments with the ITER-like Wall are not expected until 2010 it has already created a greater sense of urgency in JET task forces for development of truly ITER compatible scenarios and the design process itself is drawing out many lessons of detail which will be very relevant for ITER.

\section{References}

[Coad2001] J.P.Coad et al., J.Nucl. Mater., 290b93 (2001) 224

[Doerner2006] R.Doerner et al., "Implications of mixed material formation in ITER”, Proceedings [Hirai2006a] T. Hirai, H. Maier, M. Rubel, Ph. Mertens, et al., ”R\&D on full tungsten divertor and beryllium wall for JET ITER-like Wall Project”, submitted to Symposium on Fusion Technology, Warsaw Poland, September 2006.

[Hirai2006b] T.Hirai et al.,"Development and testing of a bulk tungsten tile for the JET divertor”, submitted to PFMCa1 Greifswald, October 2006.

[Hirai2006c] T. Hirai, J. Linke, P. Sundelin, M. Rubel, W. Kühnlein, E. Wessel, J.P. Coad, C. P. Lungu, G.F. Matthews, L. Pedrick, G. Piazza and JET-EFDA contributors, “Characterisation and Heat Flux Testing of Beryllium Coatings on Inconel”, submitted to PFMCa1 Greifswald, October 2006.

of the $17^{\text {th }}$ International Conference on Plasma Surface Interactions, Hefei, China (2006)

[Lungu2006] C.Lungu et al., "Beryllium coatings on metals: Manufacture and Characterisation”, this proceedings.

[Neu2006] R.Neu, H.Maier et al., “Development of W Coatings for JET” 
[Maier2006] H.Maier et al., “Tungsten coatings for the JET ITER-like Wall Project”, Proceedings of the $17^{\text {th }}$ International Conference on Plasma Surface Interactions, Hefei, China (2006)

[Mayer2006] M.Mayer et al., “Tungsten Erosion in the Outer Divertor of JET”, Proceedings of the $17^{\text {th }}$ International Conference on Plasma Surface Interactions, Hefei, China (2006) [Mertens2006a] Ph.Mertens et al., this proceedings.

[Mertens, 2006b] Ph.Mertens et al., "Conceptual design for a bulk tungsten divertor tile in JET" submitted to Fusion Eng. and Design (SOFT 2006 - 24th Symposium on Fusion Technology, Warsaw, Poland)

[Neu2006a] R.Neu et al., this proceedings.

[Neu2006b]R.Neu et al., "Final Steps to an All Tungsten Divertor Tokamak”, Proceedings of the $17^{\text {th }}$ International Conference on Plasma Surface Interactions, Hefei, China (2006) [Pamela2006a] J.Pamela et al., "The Contribution of JET to ITER", submitted to Fusion Eng. and Design (SOFT 2006 - 24th Symposium on Fusion Technology, Warsaw, Poland) [Pamela2006b] J.Pamela, G.F.Matthews, V.Philipps et al., “An ITER-like Wall for JET”, Proceedings of the $17^{\text {th }}$ International Conference on Plasma Surface Interactions, Hefei, China (2006)

[Nunes2006] I. Nunes et al., "Power handling of the Beryllium tiles for the ITER-like Wall project at JET”, submitted to Fusion Eng. and Design (SOFT 2006 - 24th Symposium on Fusion Technology, Warsaw, Poland)

[Thompson2006] V. Thompson, Y. Krivchenkov, V. Riccardo, Z. Vizvary, “Analysis and design of the beryllium tiles for the JET ITER-like wall project”, submitted to Fusion Eng. and Design (SOFT 2006 - 24th Symposium on Fusion Technology, Warsaw, Poland) [Watson, 1997] R.R.Watson et al., Fusion Engineeering and Design 37 (1997) 553-579

\section{Acknowledgements}

The ITER-like Wall Project has relied on staff and technical support from the following EURATOM Fusion Associations: CEA(France), FZ-Jülich (Germany), IPPGarching(Germany), MEdC(Romania), SCK-MOL(Belgium), Tekes(Finland), UKAEA(UK) and VR(Sweden). In addition we would like to thank Brush-Wellman (USA) for their help in defining procedures for beryllium machining and etching and the PISCES team (UCSD, USA) for measurements of erosion rate of beryllium coatings. This work has been carried out in the framework of the European Fusion Development Agreement and is partly funded by 
the European Communities under the Contract of Association between Euratom and UKAEA. The views and opinions do not necessarily reflect those of the European Commission. 